\title{
EMPREGO DE FONTES DE CONTAMINAÇÃO DE DIFERENTES GEOMETRIAS NAS ESTIMATIVAS DO RISCO À SAÚDE HUMANA DEVIDO À INGESTÃO DE ÁGUA SUBTERRÂNEA CONTAMINADA \\ APPLYING DIFFERENT CONTAMINATION SOURCE GEOMETRIES IN HEALTH RISK ASSESSMENT DUE TO INGESTION OF CONTAMINATED GROUNDWATER
}

\section{Tirzah Moreira Siqueira}

Professora auxiliar adjunto $\mathrm{A}$ do Centro de Engenharias da Universidade Federal de Pelotas (UFPel) - Pelotas (RS), Brasil. Doutora em Recursos Hídricos e Saneamento Ambiental pelo Instituto de Pesquisas Hidráulicas (IPH) da Universidade Federal do Rio Grande do Sul (UFRGS) - Porto Alegre (RS), Brasil.

\section{Endereço para correspondência:}

Tirzah Moreira Siqueira Universidade Federal de Pelotas 96010-020 - Pelotas (RS), Brasil E-mail: tirzahmelo@hotmail.com

Recebido: 08/08/2013

Aceito: 30/01/2017

\section{RESUMO}

A metodologia de Avaliação de Risco à Saúde Humana é comumente desenvolvida empregando a solução de Domenico para transporte de contaminantes em água subterrânea por meio de um plano vertical normal ao fluxo subterrâneo. Essa solução não se aplica a situações em que a geometria da fonte de contaminação não é um plano vertical, tais como trincheiras, poços de injeção, entre outras. Dessa forma, um conjunto de soluções analíticas para diferentes geometrias de fonte foi apresentado. Para comparação, escolheram-se as geometrias de plano vertical e prisma para verificar as influências na evolução da pluma de contaminação e suas implicações na estimativa do valor do risco de efeitos carcinogênicos por ingestão de água subterrânea contaminada por benzeno. Os resultados demonstraram não haver diferenças significativas nas estimativas da concentração de equilíbrio ao se considerar os fluxos ao longo de um plano vertical normal e através de um prisma, o que não resultou em diferenças nos riscos à saúde humana calculados a partir dessas concentrações.

Palavras-chave: geometrias de fonte de contaminação; água subterrânea; avaliação de risco à saúde humana.

\section{ABSTRACT}

Health Risk Assessment is commonly developed employing the Domenico solution for groundwater contaminant transport passing through a vertical plane normal to the groundwater flow. This solution does not apply in situations for which the contamination source geometry is not a vertical plane, such as trenches, injection wells, etc. Hence, a set of analytical solutions for different source geometries were presented. For comparison, vertical plane and prism geometries were selected to verify their influences over the contaminant plume simulations and their implications over the estimation of the risk of carcinogenic adverse effects due to groundwater ingestion contaminated by benzene. The results demonstrated small differences over the equilibrium concentration simulations, not interfering significantly in the healthy risk estimation.

Keywords: source contamination geometries; groundwater; health risk assessment. 


\section{INTRODUÇÃO}

A contaminação do solo e da água subterrânea tem recebido especial atenção nos últimos anos devido à grande quantidade de vazamentos e derrames de petróleo e seus derivados no meio ambiente. Esse tipo de contaminação representa a maior parte dos acidentes ambientais registrados no mundo (TAKEUCHI, 2008). Sobre esse fato, existe um consenso de que tais substâncias, quando presentes na água, no ar ou no solo, representam algum tipo de risco à saúde humana.

Existem muitas definições para o termo "risco"; para os fins deste estudo, cabe aqui apresentar a exposta por Kolluru (1996, p. 4.64): "a medida da probabilidade e magnitude de efeitos adversos, incluindo dano, doenças ou perdas econômicas (perigo e toxicidade não significam risco per si)", podendo o risco ser medido, controlado e gerenciado.

Dentro desse contexto, vale lembrar que grandes esforços são empregados em onerosos planos de remediação, muitas vezes ainda insuficientes para que as concentrações limites dos contaminantes aceitáveis nos meios físicos sejam atingidas. Esse fato despertou na comunidade científica a necessidade de associar aos planos de remediação uma avaliação do risco ao qual os seres humanos estão expostos, e é por esse motivo que atualmente se discute muito a avaliação de risco à saúde humana inserida em um contexto de remediação de áreas contaminadas.

O grande problema está no fato de que os aplicativos que lidam com a avaliação de risco apresentam baixa capacidade de representar as mais diversas formas de liberação e transporte de contaminantes entre os meios físicos. Além disso, não se aplicam a todos os possíveis tipos de fontes de contaminação. Como resultado, as estimativas do risco não são confiáveis ou não podem ser determinadas.

Sob o enfoque da contaminação de água subterrânea, abordado neste estudo, por exemplo, o modelo de transporte de contaminantes mais comumente utilizado é o de Domenico (1987), o qual não pode representar mais do que uma pluma de contaminação passando por um plano normal ao fluxo subterrâneo. Assim, fontes de contaminação de geometrias lineares, como um poço, ou em planos, como uma trincheira, não podem ser avaliadas em termos do risco resultante a tais for- mas de liberação de compostos, pois não existem modelos matemáticos disponíveis inseridos nesse âmbito.

Adicionalmente, Guyonnet e Neville (2004) apresentam comparações entre o modelo de Domenico (1987) e o de Sagar (1982), demonstrando que as discrepâncias entre eles são relativamente negligenciáveis ao longo do eixo central da pluma de contaminação (no sentido preferencial do fluxo), enquanto grandes para pontos que se afastam lateralmente dessa linha central, principalmente quando se considera o decaimento do contaminante. Isso sugere que os erros na solução de Domenico (1987) são significativamente influenciados pela distância lateral, possivelmente quando a dispersão e a difusão molecular influenciam o processo de transporte, e que, nesses casos, o modelo de Domenico (1987) deve ser preterido ao de Sagar (1982).

Tendo em vista a importância adquirida pela avaliação de risco à saúde humana no contexto ambiental, e as limitações que os aplicativos mais comumente utilizados para estimar o risco têm com relação aos modelos que utilizam para simular o transporte dos contaminantes em meio saturado, o presente artigo tem o objetivo de apresentar um conjunto de soluções que podem ser igualmente inseridas em avaliação de risco.

Essas soluções analíticas incorporam as mais diversas geometrias de fontes de contaminação, das quais duas foram aqui escolhidas para avaliar suas influências sobre a estimativa do risco à saúde humana, a saber: fonte com geometria de plano vertical normal ao fluxo subterrâneo e fonte prismática. As concentrações simuladas por ambas as geometrias de fonte mencionadas foram então utilizadas na predição do risco à saúde humana por meio da exposição via ingestão de água subterrânea contaminada por benzeno.

Dessa forma, com o intuito de contemplar o escopo metodológico deste artigo, inicialmente tem-se uma breve descrição referente à metodologia de avaliação de risco à saúde humana e à via de exposição que foi considerada, seguida pela apresentação do conjunto de soluções para transporte de contaminantes em meio saturado que foi empregado e um estudo de caso real aplicado a um sítio contaminado na área de uma refinaria da Região Sudeste do país. 


\section{AVALIAÇÃO DE RISCO À SAÚDE HUMANA}

A avaliação de risco, primeira etapa de um processo ainda maior denominado análise de risco, pode ser entendida como o processo de controle de situações em que um organismo, um sistema ou uma população podem estar expostos a algum perigo (WHO, 2004). Tal processo é constituído por mais dois outros além da avaliação do risco, a saber: gerenciamento e comunicação do risco.

A avaliação de risco, segundo o Conselho Nacional de Pesquisas dos Estados Unidos (NRC, 2007, p. 6), é definida como "a caracterização qualitativa ou quantitativa dos potenciais efeitos adversos à saúde humana de determinadas substâncias sobre indivíduos ou populações".

Como apresentado por vários autores (ÖRBEG \& BERGBÄCK, 2005; CARTER et al., 2003; KOLLURU, 1996; SCHLECHTER, 1995; MCCALLUM \& SANTOS, 1996), tal processo divide-se em:

- Identificação do perigo: determina-se a possibilidade de a exposição causar a incidência de efeitos adversos à saúde humana, tais como câncer. Para tanto, necessita-se da caracterização da área contaminada, dos tipos de receptores e de contaminantes, bem como das vias de exposição completas;

- Avaliação da dose-resposta: caracterização da relação entre a exposição ou a dose de um contaminante e a ocorrência de possíveis efeitos adversos à saúde, geralmente em termos de carcinogenicidade e toxicidade de um agente químico;

- Avaliação da exposição: avaliação quantitativa da intensidade, frequência e duração de um contato químico, da via de exposição (dermal, oral ou respiratória), das taxas (ingestão ou captação), da quantidade de contaminante que efetivamente atravessa as limitações corpóreas, bem como da dose interna absorvida (RICE et al., 2008);

- Caracterização do risco: etapa final que objetiva descrever a natureza e a magnitude do risco à saúde humana, por meio do cálculo do risco e das concentrações limites aceitáveis dos contaminantes para cada combinação de meio de contato, via de exposição e tipo de receptor (NCR, 1983).

O gerenciamento do risco é definido como o processo de valoração e, se necessário, controle das fontes de exposição e risco (KOLLURU, 1996). Isso implica na escoIha de ações regulatórias que melhor se apliquem sobre o sítio contaminado, visando à redução ou à eliminação do risco. Tais ações estão diretamente baseadas nos resultados obtidos no processo de avaliação do risco.

E, por fim, o processo de comunicação do risco representa a troca de informações entre as partes interessadas (agências governamentais, corporações ou grupos industriais, sindicatos, meio científico, organizações profissionais, etc.) sobre a natureza, a magnitude, a significância ou o controle de um risco por meio de uma variedade de canais de comunicação (COVELLO, 1996).

Todas essas etapas estão inter-relacionadas e não podem ser vistas como processos independentes uns dos outros.

A metodologia mais utilizada para avaliação de risco à saúde humana foi desenvolvida pela American Society of Testing and Materials (ASTM), inicialmente elaborada para hidrocarbonetos e posteriormente estendida a outras substâncias. A metodologia, denominada Risk Based Corrective Action (RBCA), traduzida como Ação Corretiva Baseada no Risco, tem sido largamente aplicada e já é aceita em muitos países como instrumento de legislação ambiental.

\section{VIA DE EXPOSIÇÃO AO RISCO}

A aplicação da metodologia RBCA requer que sejam avaliadas as vias de exposição ao receptor que estejam completas. Isso equivale a dizer que devem ser avaliadas as vias de exposição para as quais tem-se uma fonte de contaminação, um meio e mecanismo de transporte dos contaminantes e um receptor. Para o desenvolvimento do estudo de caso apresentado no presente artigo, considerou-se a via de exposição por ingestão de água subterrânea contaminada para contato do receptor com o contaminante.

Apenas o risco para efeitos carcinogênicos do benzeno foi avaliado, a título de ilustração do emprego das soluções analíticas adiante apresentadas. 
A Equação 1 define o risco para essa via de exposição e é dada por (USEPA, 1989):

$$
R=\frac{C_{P O E} \times C R \times E F \times E D}{B W \times A T} \times S F
$$

Em que:

$\mathrm{R}$ é o risco de efeitos carcinogênicos (adimensional); $\mathrm{C}_{\mathrm{POE}}$ é a concentração do contaminante no ponto de exposição $(\mathrm{mg} / \mathrm{L})$;

CR é a taxa de ingestão de água subterrânea (L/dia);

EF é a frequência de exposição (dias/ano);

ED é a duração da exposição (anos);

BW é a massa corpórea $(\mathrm{kg})$;

AT é o tempo de exposição média (dias); e

SF é o fator de carcinogenicidade (kg.dia/mg).

Considerando a $C_{P O E}$ e que, em geral, as concentrações são amostradas apenas na fonte, a determinação da concentração no ponto de exposição é feita por meio da Equação 2, como segue:

$C_{\text {POE }}=C \times D A F$
Em que:

C é a concentração na fonte de contaminação (mg/L); e DAF é o fator de atenuação por dispersão lateral de um contaminante (-).

O termo DAF cobre todos os processos físicos, químicos e biológicos que contribuem para a redução da massa de contaminantes dissolvidos na pluma de contaminação, por meio de diversos processos, tais como advecção, dispersão, biodegradação, adsorção, volatilização, entre outros (BATLE-AGUILAR et al., 2009). Para tanto, na determinação desse fator de atenuação se faz necessária a utilização de modelos de transporte de contaminantes que predigam qual será a concentração em um determinado ponto no aquífero, para um instante $t$ também especificado.

A seguir é apresentado um conjunto de soluções analíticas para transporte de contaminantes em diferentes geometrias de fonte. A partir dessas soluções será possível determinar as concentrações de benzeno no ponto de exposição e, consequentemente, o risco que ele oferece ao contato com receptores potenciais, considerando as geometrias de plano vertical normal ao fluxo e prisma.

\section{SOLUÇÕES ANALÍTICAS PARA O TRANSPORTE EM ÁGUA SUBTERRÂNEA}

Encontra-se na literatura uma série de trabalhos que apresentam soluções analíticas para o transporte de contaminantes em meio saturado partindo de diferentes geometrias de fonte. Sim e Chrysikopoulos (1999) apresentaram soluções analíticas para a incomum fonte de geometria elíptica localizada em um plano horizontal, considerando aquíferos de espessuras finita e infinita.

Levando em consideração as dispersões radial e axial (profundidade), Massabó, Cianci e Paladino (2006) determinaram soluções bidimensionais para uma fonte de geometria cilíndrica, cuja principal aplicação é a determinação dos coeficientes de dispersão longitudinal e transversal ao mesmo tempo, o que seria muito difícil de se obter em campo.

Merece destaque especial o trabalho de Leij, Priesack e Schaap (2000), em que são apresentadas mais de dez funções de Green para diferentes condições de contorno e problemas de valor inicial em meios porosos. Tais funções permitem que uma ampla gama de situações possa ser resolvida considerando, também, diferentes geometrias de fonte. Trabalho semelhante foi desenvolvido por Park e Zhan (2001) para soluções analíticas de transporte de contaminantes partindo de fontes de contaminação pontuais, linhas, planos e volumes. Esse trabalho serviu para comparação das soluções apresentadas a seguir.

As soluções analíticas aqui apresentadas estão divididas em dois grupos, associados ao tipo de liberação do contaminante na fonte: instantânea ou contínua, conforme apresentado em Melo (2010).

A Equação 3 é a equação diferencial parcial para transportes de contaminantes em meio saturado e fluxo uniforme, considerando sorção instantânea, linear e reversível, bem como decaimento de primeira ordem do contaminante, e dada sob a forma:

$$
\frac{D_{x}}{R} \frac{\partial^{2} C}{\partial x^{2}}+\frac{D_{y}}{R} \frac{\partial^{2} C}{\partial y^{2}}+\frac{D_{z}}{R} \frac{\partial^{2} C}{\partial z^{2}}-\frac{v}{R} \frac{\partial C}{\partial x}-\mu C=\frac{\partial C}{\partial t}
$$


Em que:

C é a concentração do contaminante $\left[\mathrm{ML}^{-3}\right]$;

$R$ é o fator de retardo do contaminante [-];

$D_{x}$ é o coeficiente de dispersão longitudinal $\left[L^{2} T^{-1}\right]$;

$D_{y}$ e $D_{z}$ são os coeficientes de dispersão transversal $\left[L^{2} T^{-1}\right]$;

$x, y$ e $z$ são as coordenadas do ponto de exposição [L];

$\mu$ é a constante de decaimento de primeira ordem do contaminante $\left[\mathrm{T}^{-1}\right]$; e

t é o tempo [T].

Para a condição de contorno, a fonte é caracterizada por uma determinada "massa" de entrada, o que pode ocorrer em um ponto, ao longo de uma linha, sobre um plano ou por meio de um volume. A Equação (3) foi dividida em três equações unidimensionais $(4,5$ e 6), cujas soluções estão representadas pelas Equações 7, 8 e 9:

$\frac{D_{x}}{R} \frac{\partial^{2} f_{1}}{\partial x^{2}}-\frac{v}{R} \frac{\partial f_{1}}{\partial x}-\mu f_{1}-\frac{\partial f_{1}}{\partial t}=0$

$$
\frac{D_{y}}{R} \frac{\partial^{2} f_{2}}{\partial y^{2}}-\frac{\partial f_{2}}{\partial t}=0
$$

\section{Liberação instantânea de contaminante}

Uma entrega instantânea de contaminante consiste na liberação de uma concentração (ou massa) fixa por um curto período de tempo. Partindo da

\section{Pontual}

$C(x, y, z, t)=\frac{M_{0}}{\varphi R} f_{1}\left(x-x^{\prime}, t\right) \cdot f_{2}\left(y-y^{\prime}, t\right) \cdot f_{3}\left(z-z^{\prime}, t\right)$

\section{Linhas}

A liberação instantânea de massa em uma linha pode ser obtida pela aproximação de uma sequência de pontos muito próximos. Para esse caso, tem-se então uma quantidade de massa liberada por unidade de comprimento de linha da fonte $\left(M_{1}\right)$ (Figura 1).

$$
\begin{aligned}
& \frac{\mathrm{D}_{\mathrm{z}}}{\mathrm{R}} \frac{\partial^{2} \mathrm{f}_{3}}{\partial \mathrm{z}^{2}}-\frac{\partial \mathrm{f}_{3}}{\partial \mathrm{t}}=0 \\
& \mathrm{f}_{1}(\mathrm{x}, \mathrm{t})=\frac{1}{2\left(\pi D_{x} t / R\right)^{1 / 2}} \exp \left\{\left[-(\mathrm{x}-(\mathrm{vt} / \mathrm{R}))^{2} /\left(4 D_{x} t / R\right)\right]-\mu t\right\} \\
& \mathrm{f}_{2}(\mathrm{y}, \mathrm{t})=\frac{1}{2\left(\pi \mathrm{D}_{\mathrm{y}} \mathrm{t} / \mathrm{R}\right)^{1 / 2}} \exp \left\{-\mathrm{y}^{2} /\left(4 \mathrm{D}_{\mathrm{y}} \mathrm{t} / \mathrm{R}\right)\right\} \\
& \mathrm{f}_{3}(\mathrm{z}, \mathrm{t})=\frac{1}{2\left(\pi \mathrm{D}_{\mathrm{z}} \mathrm{t} / \mathrm{R}\right)^{1 / 2}} \exp \left\{-\mathrm{z}^{2} /\left(4 \mathrm{D}_{\mathrm{z}} \mathrm{t} / \mathrm{R}\right)\right\}
\end{aligned}
$$

Assim, o produto $f_{1} \cdot f_{2} \cdot f_{3}$ satisfaz a Equação (3), o que pode ser facilmente verificado. $\mathrm{O}$ balanço de massa da Equação (3) por ser mantido por:

$$
\varphi R \int_{-\infty}^{\infty} f_{1} d x \int_{-\infty}^{\infty} f_{2} d y \int_{-\infty}^{\infty} f_{3} d z=M_{\circ}
$$

Em que:

$\phi$ é a porosidade do aquífero [-];

$\mathrm{R}$ é o fator de retardo [-]; e $\mathrm{M}_{\mathrm{o}}$ é a massa liberada no aquífero [M].

Equação (10), as soluções para as diversas geometrias de fonte podem ser dadas pelos casos subsequentes.

Em que:

$\mathrm{M}_{\mathrm{o}}$ é a massa liberada para o aquífero; e

$\left(x^{\prime}, y^{\prime}, z^{\prime}\right)$ é o ponto de liberação de contaminante.

Um exemplo desse tipo de fonte é um poço de injeção cujo contato com o aquífero se estende por todo o filtro.

Aplicando-se a Equação (11), a soma das respostas individuais é a resposta da liberação de massa distribuída ao longo da linha. A Equação (10), para esse caso, torna-se: 
$C=\frac{M_{1}}{\varphi R} \int_{-\infty}^{\infty} f_{1}\left(x-x^{\prime}, t\right) \cdot f_{2}\left(y-y^{\prime}, t\right) \cdot f_{3}\left(z-z^{\prime}, t\right) d z^{\prime}$

Para o caso de uma linha finita, a Equação (12) passa a ser integrada sobre o intervalo finito:

$\int_{-L_{3}}^{L_{3}} f_{3}\left(z-z^{\prime}, t\right) d z=F_{3}=\frac{1}{2}\{\operatorname{erf}$

$\left[\left(z+L_{3}\right) /\left(2\left(D_{z} t / R\right)^{1 / 2}\right)\right]-\operatorname{erf}\left[\left(z-L_{3}\right) /\left(2\left(D_{z} t / R\right)^{1 / 2}\right]\right\}$

O resultado final para uma linha finita paralela a $z$ e localizada em $x^{\prime}=y^{\prime}=0$ é:

$C(x, y, z, t)=\frac{M_{1}}{\varphi R} f_{1}(x, t) \cdot f_{2}(y, t) \cdot F_{3}(z, t)$

A solução para uma linha de comprimento finito, paralela a y, e localizada em $x^{\prime}=z^{\prime}=0$ é dada pela Equação
(15), em que $F_{2}$ é diferente de $F_{3}$ apenas pela substituição de $L_{3}$ por $L_{2}$, e de $z$ por $y$ :

$C(x, y, z, t)=\frac{M_{1}}{\varphi R} f_{1}(x, t) \cdot F_{2}(y, t) \cdot f_{3}(z, t)$

Finalmente, para o caso de uma linha de comprimento finito, paralela ao eixo x e localizada em $y^{\prime}=z^{\prime}=0$ :

$C(x, y, z, t)=\frac{M_{1}}{\varphi R} F_{1}(x, t) \cdot f_{2}(y, t) \cdot f_{3}(z, t)$

Em que:

$$
\begin{aligned}
& F_{1}(x, t)=\frac{1}{2}\left\{\operatorname{erf}\left[\left(x+L_{1}-v t\right) /\left(2\left(D_{x} t / R\right)^{1 / 2}\right)\right]\right. \\
& -\operatorname{erf}\left[\left(x-L_{1}-v t\right) /\left(2\left(D_{x} t / R\right)^{1 / 2}\right]\right\}
\end{aligned}
$$

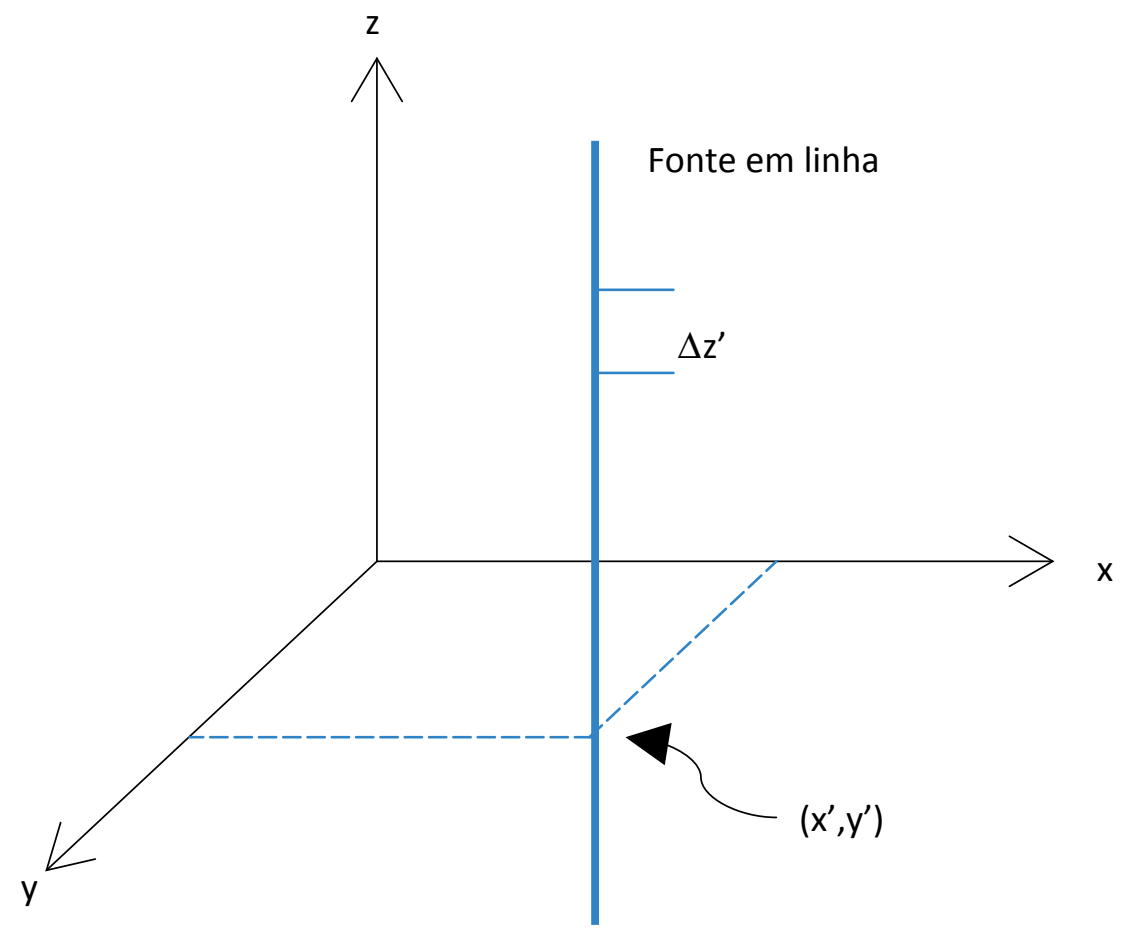

Figura 1 - Geometria de fonte em linha. 


\section{Planos}

No caso anterior a solução foi obtida pela superposição de uma série de pontos ao longo de uma linha. No caso do plano, tem-se a distribuição de linhas sobre um plano finito de dimensões $2 L_{2} \times 2 L_{3}$, no qual $M_{2}$ é a massa liberada por unidade de área do aquífero (Figura 2).

As soluções para planos paralelos aos eixos coordenados são:

Paralelo ao eixo yz:

$C(x, y, z, t)=\frac{M_{2}}{\varphi R} f_{1}(x, t) \cdot F_{2}(y, t) \cdot F_{3}(z, t)$

\section{Volume}

Para esse caso, o termo $M_{3}$ é a massa liberada por unidade de volume de meio poroso dentro de um prisma com dimensões $2 \mathrm{~L}_{1} \times 2 \mathrm{~L}_{2} \times 2 \mathrm{~L}_{3}$, tendo como centro das coordenadas o centro do prisma, e a distribuição de concentração do contaminante é dada por:
Paralelo ao eixo xz:

$C(x, y, z, t)=\frac{M_{2}}{\varphi R} F_{1}(x, t) \cdot f_{2}(y, t) \cdot F_{3}(z, t)$

Paralelo ao eixo xy:

$$
C(x, y, z, t)=\frac{M_{2}}{\varphi R} F_{1}(x, t) \cdot F_{2}(y, t) \cdot f_{3}(z, t)
$$

$C(x, y, z, t)=\frac{M_{3}}{\varphi R} F_{1}(x, t) \cdot F_{2}(y, t) \cdot F_{3}(z, t)$

A Figura 3 traz a representação esquemática para o caso de uma fonte de volume definido, considerando que o prisma pode assumir qualquer posição dentro do aquífero.

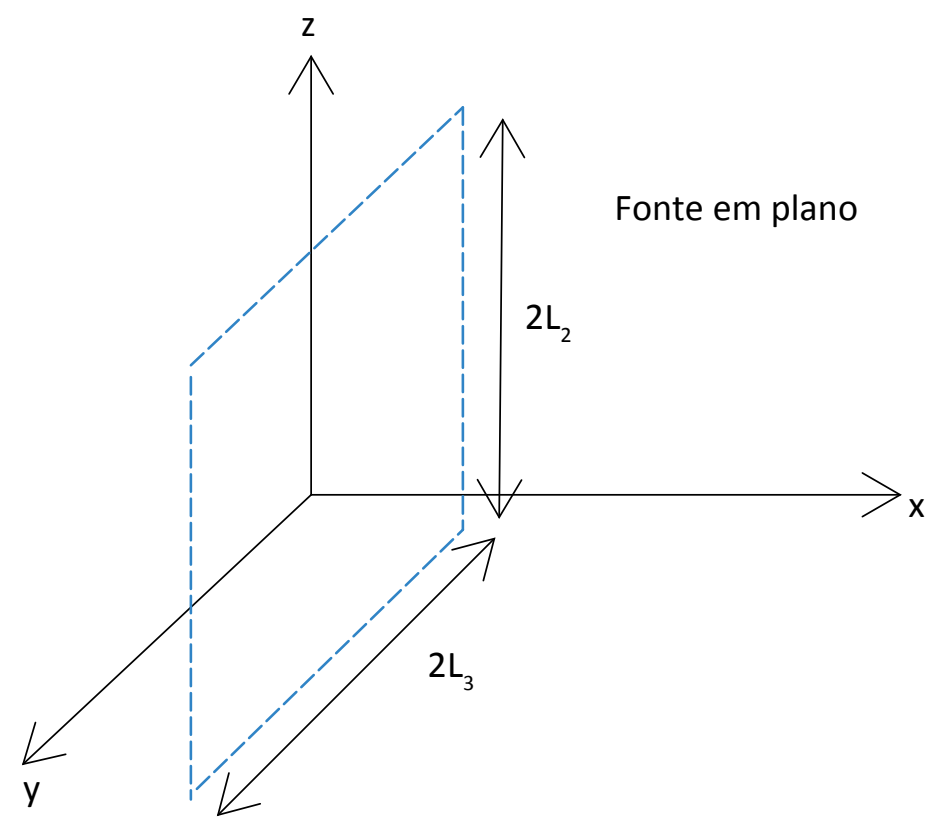

Figura 2 - Geometria de fonte em plano. 


\section{Liberação contínua de contaminante}

No caso da liberação contínua de contaminantes, a entrega de massa pode não variar com o tempo; pelo contrário, pode se prolongar consideravelmente. Outras situações possíveis são: a concentração variar irregularmente com o tempo, ou a entrega de massa diminuir com o tempo. O entendimento desse tipo de fonte pode ser obtido partindo-se do caso de uma fonte pontual, e substituindo nela $t$ por $(\mathrm{t}-\tau)$, em que $\tau$ representa o tempo decorrido desde o momento da entrega de massa em qualquer ponto de interesse. A distribuição de concentrações resultante no tempo $\tau$ é:

$C(x, y, z, t)=\frac{M_{o}}{\varphi R} f_{1}(x, t-\tau) \cdot f_{2}(y, t-\tau) \cdot f_{3}(z, t-\tau)$

Devido à linearidade da equação, pode-se aproximar a resposta a uma liberação contínua, considerando a sobreposição de uma sequência de liberações instantâneas muito próximas. Supondo-se que tenha ocorrido uma liberação contínua durante um intervalo de tempo muito pequeno, $\Delta \tau$, centrado em $\tau$, então a Equação (22) pode ser utilizada para a obtenção da resposta, admitindo-se:
$M_{o}=\frac{\Delta M_{o}}{\Delta \tau} \Delta \tau$

Devido a uma liberação contínua no tempo e que representa a adição de respostas de cada liberação instantânea, a resposta referente à Equação (22) aparecerá na forma discreta como:

$C(x, y, z, t-\tau)=\frac{1}{\varphi R} \sum_{i=1}^{n} \frac{\Delta M_{o}}{\Delta \tau} \mid \tau_{i} \Delta \tau f_{1}(x, t-\tau) \cdot f_{2}(y, t-\tau) \cdot f_{3}(z, t-\tau)(24)$

Considerando-se $\Delta \tau \rightarrow 0$, obtém-se:

$C(x, y, z, t-\tau)=\frac{1}{\varphi R} \int_{0}^{t} \frac{d M_{0}}{d \tau} f_{1}(x, t-\tau) \cdot f_{2}(y, t-\tau) \cdot f_{3}(z, t-\tau) d \tau$

Essa equação foi obtida exclusivamente para o caso de uma fonte pontual. Além disso, se a taxa de liberação de massa for constante, o termo $\mathrm{dM}_{\mathrm{o}} / \mathrm{d} \tau$ pode ser pos-

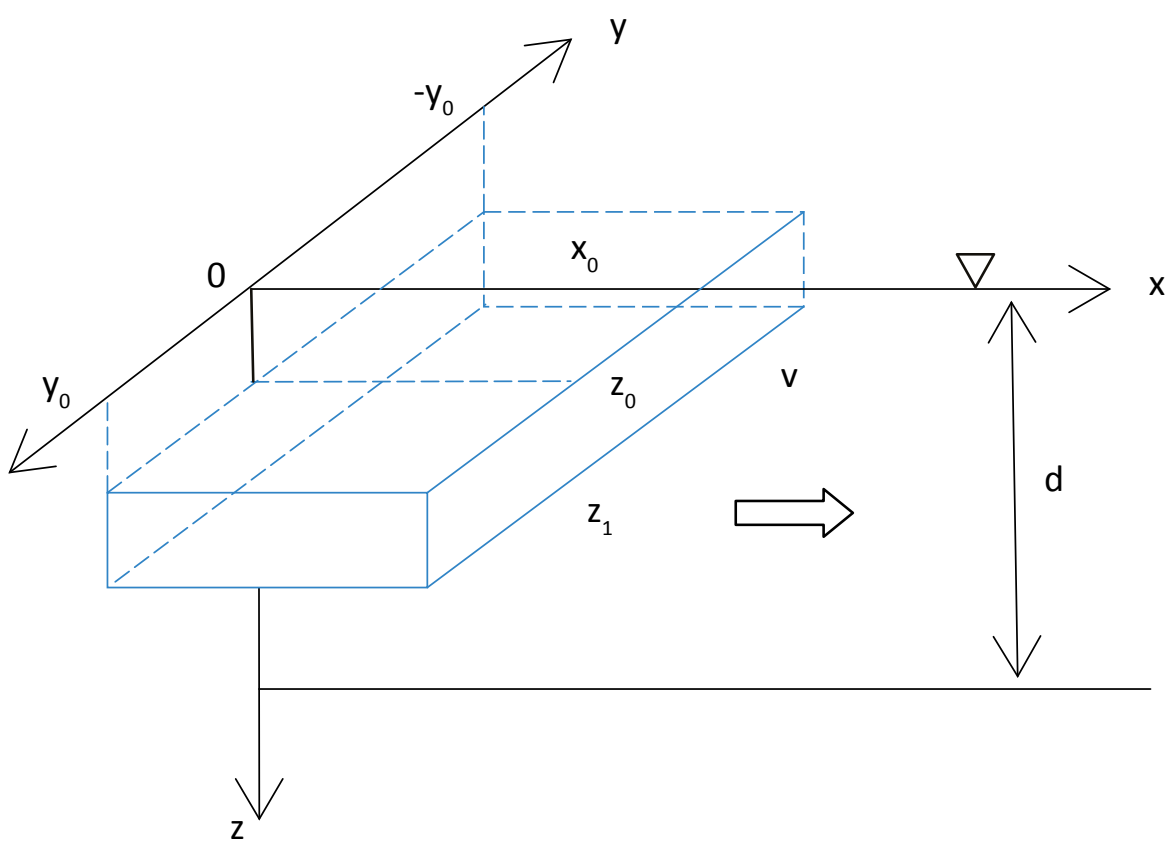

Figura 3 - Geometria de fonte com volume definido. 
to fora da integral. De forma mais geral, permitindo a aplicação de qualquer geometria da fonte, tem-se:

$C(x, y, z, t-\tau)=\int_{0}^{t} \frac{d M_{n}}{d \tau} K_{n}(x, y, z, t-\tau) \cdot d \tau$

Pontual

$M_{n}=M_{o}=$ massa liberada;

\section{Linhas}

$M_{n}=M_{1}=$ massa liberada por unidade de comprimento;

$K_{1}=\frac{1}{\varphi R} F_{1} \cdot f_{2} \cdot f_{3}$, paralela ao eixo $x$

\section{Planos}

$M_{n}=M_{2}=$ massa liberada por unidade de área;

$K_{2}=\frac{1}{\varphi R} F_{1} \cdot F_{2} \cdot f_{3}$, plano horizontal paralelo ao eixo $x y$;
O termo $\mathrm{K}_{\mathrm{n}}$ representa a resposta do sistema a uma entrega instantânea unitária, isto é, é a resposta do sistema a "impulsos unitários" sobre fontes de diferentes geometrias. Abaixo estão listados os termos $K_{n}$ e $M_{n}$ para cada tipo de fonte.

$K_{o}=\frac{1}{\varphi R} f_{1} \cdot f_{2} \cdot f_{3}$.

$\mathrm{K}_{1}=\frac{1}{\varphi \mathrm{R}} \mathrm{f}_{1} \cdot \mathrm{F}_{2} \cdot \mathrm{f}_{3}$, paralela ao eixo $\mathrm{y}$;

$K_{1}=\frac{1}{\varphi R} f_{1} \cdot f_{2} \cdot F_{3}$, paralela ao eixo $z$

$K_{2}=\frac{1}{\varphi R} f_{1} \cdot F_{2} \cdot F_{3}$, plano horizontal paralelo ao eixo $y z ;$

$K_{2}=\frac{1}{\varphi R} F_{1} \cdot F_{2} \cdot f_{3}$, plano horizontal paralelo ao eixo $x z$.

\section{Prisma}

$M_{n}=M_{3}=$ massa liberada por unidade de volume;

$\mathrm{K}_{3}=\frac{1}{\varphi R} \mathrm{~F}_{1} \cdot \mathrm{F}_{2} \cdot \mathrm{F}_{3}$, prisma.

\section{ESTUDO DE CASO}

\section{Descrição da área contaminada}

A área de interesse consiste em um antigo depósito de resíduos oleosos provenientes das atividades de uma refinaria localizada na Região Sudeste do país, cuja identificação não será divulgada por motivos contratuais. Há 22 anos a área não é mais utilizada para depositar esses resíduos. Porém, análises químicas indicaram a existência de contaminação por hidrocarbonetos de petróleo e metais.

Os solos da área natural da refinaria foram caracterizados como sendo de moderada a alta capacidade de infiltração, a qual varia de 40 a $80 \mathrm{~mm} / \mathrm{h}$, representando a capacidade de recarga do aquífero. No entanto, na área do depósito foi detectado que a superfície é praticamente impermeável devido à formação de crostas endurecidas de resíduos oleosos.

A condutividade hidráulica é o parâmetro hidrogeológico mais importante dentro de um contexto de contaminação de água subterrânea, pois determina como o contaminante se desloca com a pluma de contaminação. Para a área fora do depósito, os valores medidos de 0,30 a $0,85 \mathrm{~m} /$ dia representam uma capacidade de movimentação lateral da água subterrânea de baixa a moderada.

Todas essas informações são importantes para analisar o comportamento do deslocamento da pluma de con- 
taminação advinda do depósito de resíduos oleosos. Para tanto, devido ao seu alto potencial carcinogênico,

\section{Simulação da concentração de benzeno}

Para a realização da simulação do transporte de contaminantes em meio saturado segundo as equações descritas e determinação da concentração de benzeno no ponto de exposição, o programa Transporte de Contaminantes em Meio Saturado (TCMS) foi desenvolvido em linguagem do aplicativo MATLAB ${ }^{\circledR}$.

Os resultados da modelagem computacional foram validados por comparação com os resultados das soluções analíticas propostas por Park e Zhan (2001) (Figura 4). As soluções foram obtidas para o ponto $(x, y, z)=(50 \mathrm{~m}, 0,0), v=0,5 \mathrm{~m} /$ dia; $D_{x}=0,25 \mathrm{~m}^{2} /$ dia, $D_{y}=D_{z}=0,005 \mathrm{~m}^{2} / \mathrm{dia} ; \mathrm{q}=3333,33$ (g/unidade de comprimento área ou volume); $\mathrm{d}=10 \mathrm{~m}$ (espessura do aquífero); $\mu=0 ; R=1, \phi=0,3$.

É possível observar a instabilidade numérica no modelo de Park e Zhan (2001), o qual oscila para altos valores da variável tempo, embora seja possível verificar qual é a concentração de equilíbrio. As comparações demonstram a semelhança entre os modelos e sua validade na simulação do transporte de contaminantes em meio saturado.

Para o estudo de caso real, as geometrias de fonte escolhidas foram o prisma e o plano vertical normal ao fluxo subterrâneo - mesmo caso em que se aplica a solução de Domenico (1987) —, a fim de que fosse possível comparar as implicações da escolha de uma ou outra.

Os dados utilizados na simulação do transporte de benzeno em meio saturado estão apresentados no Quadro 1. Para que fosse possível comparar as geometrias de fonte de plano vertical e prisma, a degradação do contaminante não foi considerada, uma vez que a equação que representa a geometria de prisma foi desenvolvida sem o termo correspondente ao decaimento de primeira ordem, provavelmente por motivos de simplificações no modelo (ver Equações 7 e 17 para os termos $f_{1}$ e $F_{1}$ ).

Na prática, uma suposição muito comum é considerar o contaminante se movimentando com a mesma velocidade da água subterrânea, isto é, $R=1$. De fato, dentro de um contexto de avaliação de risco à saúde humana, o benzeno foi utilizado para a aplicação da metodologia apresentada até aqui.

tal suposição pode ser aceitável, uma vez que contempla uma situação mais conservadora, implicando que se assume um nível de segurança maior na estimativa do risco. Dessa forma, se não houver risco segundo uma suposição de $R=1$, também não haverá risco para nenhum outro valor de retardo.

Adicionalmente, a concentração de benzeno foi tomada como a média dos valores obtidos em amostragens sobre toda a área horizontal da fonte e a diferentes profundidades. O objetivo dessas simulações é determinar as concentrações de equilíbrio do benzeno, considerando as geometrias de plano vertical e prisma, para que sejam quantificadas as estimativas do risco de câncer por ingestão de água subterrânea contaminada por essa substância, verificando as influências de se escolher uma fonte de geometria em plano vertical ou prisma sobre o valor do risco à saúde humana.

As simulações resultantes estão apresentadas na Figura 5.

As concentrações simuladas pelo prisma são maiores do que as geradas pelo plano, como era esperado; as do plano são maiores que para linhas; e estas, consequentemente, são maiores que em casos de fontes pontuais. Ambas as curvas apresentaram comportamento sem instabilidade aparente na solução. Foram necessários 400 anos de simulação devido, principalmente, à baixa velocidade de percolação e à condutividade hidráulica consideradas.

O fato de a pluma de contaminação levar tempo para chegar ao ponto de exposição exige ainda mais atenção por parte dos tomadores de decisões, pois não se pode prever o uso futuro da área, principalmente quando se tem conhecimento de que os contaminantes ainda retidos na matriz do solo podem atingir o ponto de interesse centenas de anos após o início da contaminação. Outro fator interessante é que, para os dois casos analisados, os tempos para atingir a situação de equilíbrio são muito semelhantes. Para o caso do plano, observa-se que a concentração de equilíbrio começa a ser atingida por volta de 200 anos, enquanto para o caso do prisma isso acontece após aproximadamente 250 anos. 


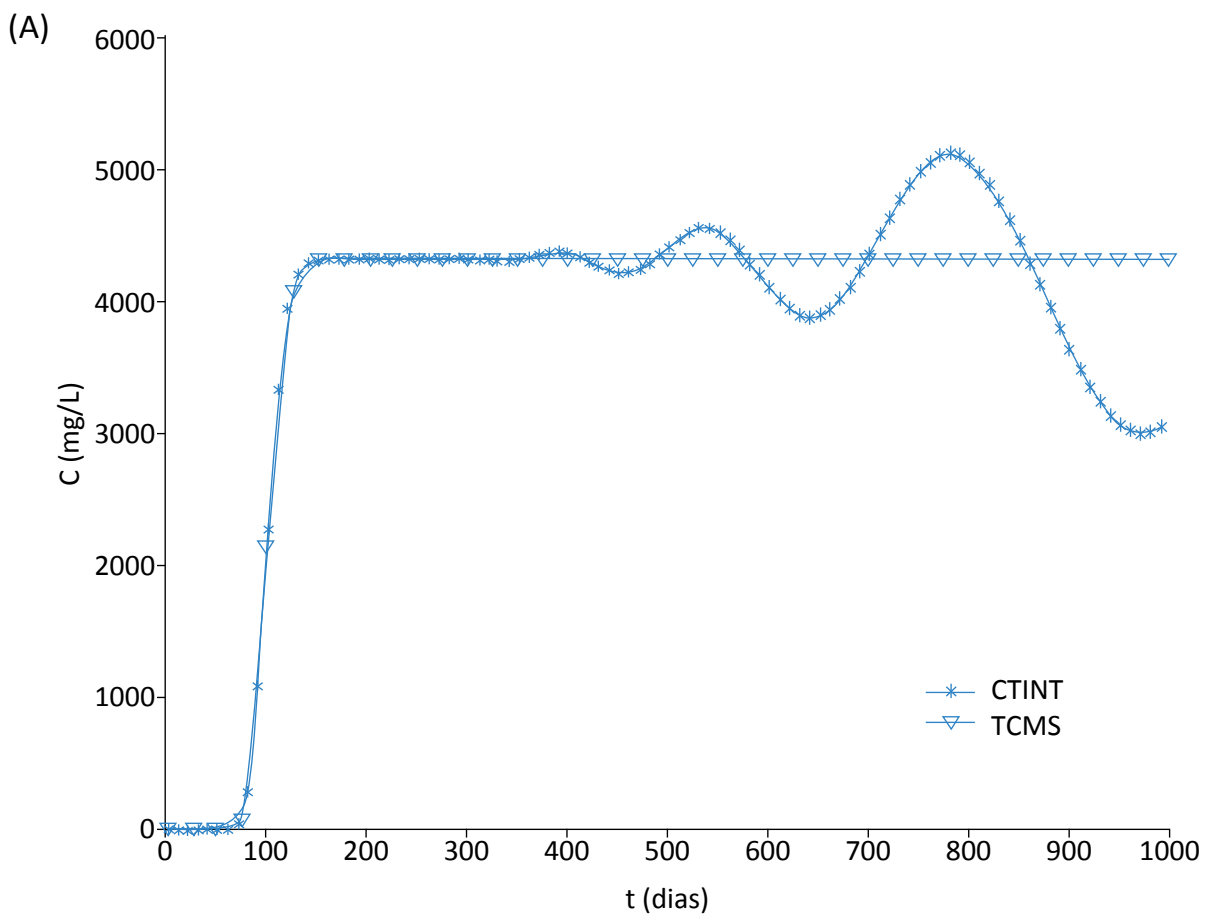

(B)

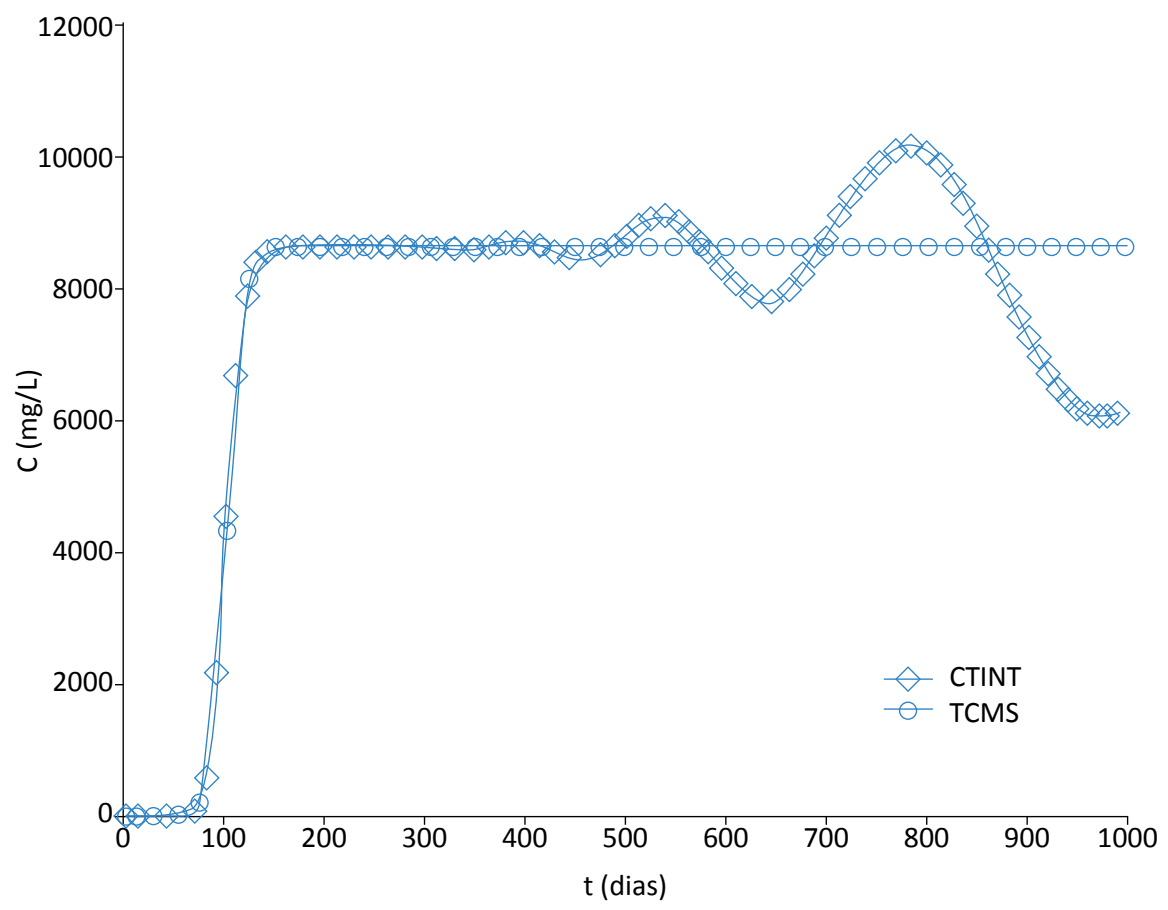

C: concentração do contaminante no ponto de exposição; TCMS: Transporte de Contaminantes em Meio Saturado.

Figura 4 - Validação do modelo Transporte de Contaminantes em Meio Saturado em comparação com o modelo CTINT de Park e Zhan (2001) e de Domenico (1987) em (A) para a geometria de plano vertical e em (B) para o prisma. 
Essa diferença pode ser atribuída à forma como os contaminantes começam a se dispersar na fonte de contaminação. Enquanto no prisma a dispersão se dá ao longo de todo o comprimento da fonte (dimensão $X$, que não há no caso do plano), no plano vertical isso não ocorre.
As concentrações de equilíbrio correspondentes para essas geometrias de fonte foram: 2,00E-05 mg/L (pris$\mathrm{ma}$ ) e $1,00 \mathrm{E}-05 \mathrm{mg} / \mathrm{L}$ (plano vertical). Utilizaram-se as concentrações de equilíbrio porque elas correspondem ao máximo risco possível de ocorrer.

\section{Determinação dos riscos de câncer via ingestão de água contaminada por benzeno}

Para a determinação dos riscos de câncer por ingestão de água subterrânea contaminada, foram considerados receptores dos tipos "comerciais" e "trabalhadores eventuais", os quais estão expostos por diferentes períodos de tempo ao benzeno. Os dados necessários para a aplicação das Equações (1) e (2) mencionadas anteriormente estão listados no Quadro 2.

Para o caso do plano vertical, o risco de efeitos carcinogênicos por ingestão de água subterrânea contaminada por benzeno foi de 4,30E-09 e 1,24E-10 para receptores dos tipos "comercial" e "trabalhadores eventuais", respectivamente. Na situação em que foi considerada a geometria de prisma, os riscos foram 8,60E-09 e 2,48E-10, para receptores "comerciais" e "trabalhadores eventuais", respectivamente. Como risco limite para todas as geometrias sugeriu-se 1,00E-06 ( 1 caso adicional de câncer em 1.000 .000 de pessoas expostas), indicando que não existe risco de câncer por exposição ao benzeno para esses tipos de receptores expostos nas frequências consideradas.

Adicionalmente, os riscos obtidos pelo prisma não são significativamente diferentes daqueles obtidos pela simulação do plano vertical, embora sejam maiores. Isso não implica, no entanto, que o prisma seja a escolha mais adequada, uma vez que apenas as considerações de não degradação e retardo nulo foram assumidas. Isso significa dizer que em casos menos conservadores, para os quais seja importante considerar, por exemplo,

Quadro 1 - Dados utilizados para a simulação da concentração do benzeno.

\begin{tabular}{|c|c|c|}
\hline Parâmetro & Valor & Descrição \\
\hline$(x, y, z)(m)$ & $(200,0,0)$ & Ponto de exposição do receptor. \\
\hline t (dias) & 400 anos & Tempo de simulação após o início da contaminação. \\
\hline$\alpha_{x}(m)$ & 40 & Dispersividade longitudinal estimada pela equação. \\
\hline$\alpha_{y}(m)$ & 13 & Dispersividade transversal estimada pela equação. \\
\hline$\alpha_{z}(m)$ & 2 & Dispersividade transversal estimada pela equação. \\
\hline$D_{x}\left(m^{2} / d i a\right)$ & 0,26 & Coeficiente de dispersão longitudinal estimado por $D_{x}=v \cdot \alpha_{x}$. \\
\hline$D_{y}\left(m^{2} / d i a\right)$ & 0,08 & Coeficiente de dispersão transversal estimado por $D_{y}=v \cdot \alpha_{y}$. \\
\hline $\mathrm{D}_{\mathrm{z}}\left(\mathrm{m}^{2} / \mathrm{dia}\right)$ & 0,01 & Coeficiente de dispersão transversal estimado por $D_{z}=v . \alpha_{z}$. \\
\hline$v(\mathrm{~m} / \mathrm{dia})$ & 0,0064 & Velocidade do fluxo subterrâneo estimado por v=q/f. \\
\hline$q(\mathrm{~m} / \mathrm{dia})$ & 0,0012 & Descarga específica do aquífero ou velocidade de Darcy. \\
\hline$f_{o c}(-)$ & 0,01 & Fração de carbono orgânico. \\
\hline$\phi(-)$ & 0,19 & Porosidade efetiva medida no campo. \\
\hline$X(m)$ & 15 & Comprimento da fonte na direção $x$. \\
\hline$Y(m)$ & 10 & Largura da pluma na fonte medida do mapa. \\
\hline $\mathrm{Z}(\mathrm{m})$ & 3 & Espessura da pluma na fonte. \\
\hline $\mathrm{R}(-)$ & 1 & Retardo do contaminante. \\
\hline$\mu(1 /$ dia $)$ & 0 & Sem decaimento de primeira ordem do benzeno. \\
\hline $\mathrm{C}_{\mathrm{o}}(\mathrm{mg} / \mathrm{L})$ & 0,0019 & Concentração de benzeno em água subterrânea. \\
\hline
\end{tabular}




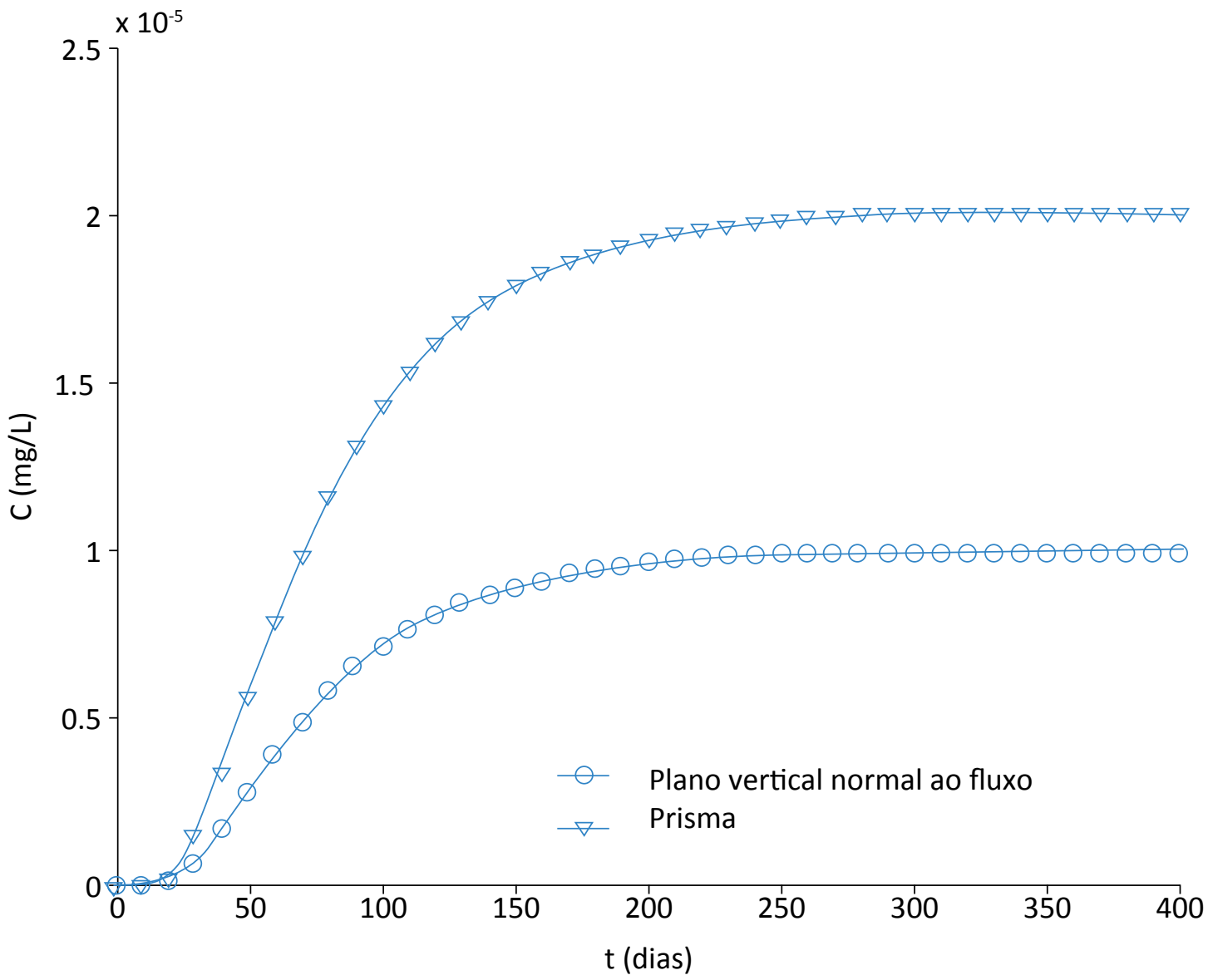

Figura 5 - Comparação entre as geometrias de fonte de plano vertical normal ao fluxo e prisma.

Quadro 2 - Dados do benzeno para cálculo do risco de câncer para via de exposição por ingestão de água subterrânea.

\begin{tabular}{|l|c|c|}
\multirow{2}{*}{ Parâmetro } & \multicolumn{2}{|c|}{ Valor } \\
\cline { 2 - 3 } & Comerciais & Trabalhadores eventuais \\
\hline AT (anos) & 68 & 68 \\
\hline ED (anos) & 25 & 1 \\
\hline EF (dias/ano) & 250 & 180 \\
\hline BW (kg) & 70 & 70 \\
\hline$C_{\text {POE }}$ (mg/L) plano vertical & $1,00 E-05$ & $1,00 E-05$ \\
\hline$C_{\text {POE }}$ (mg/L) prisma & $2,00 E-05$ & $2,00 E-05$ \\
\hline SF (kg.dia/mg) & 0,0299 & 0,0299 \\
\hline CR (L/dia) & 4 & 4 \\
\hline
\end{tabular}

AT: tempo de exposição média (dias); ED: duração da exposição (anos); EF: frequência de exposição (dias/ano); BW: massa corpórea (kg); $C_{P O E}$ : concentração do contaminante no ponto de exposição (mg/L); SF: fator de carcinogenicidade (kg.dia/mg); CR: taxa de ingestão de água subterrânea (L/dia). 
a forte tendência que um contaminante tem de se degradar, o prisma não pode ser aplicado, tendo em vista que as equações utilizadas não consideram essa característica no transporte do contaminante.

No entanto, dentro do contexto do conservadorismo adotado pela metodologia RBCA e visando a um nível de segurança maior na predição do risco, a geometria de prisma pode ser utilizada na estimativa de um risco cujo nível de segurança é ainda maior que no caso do plano vertical, uma vez que a concentração de equilíbrio é maior para aquele do que para este.

Outra consideração importante é que os riscos foram calculados empregando-se as concentrações de má- xima exposição. Isso revela a importância de que a concentração de um contaminante seja simulada até seu estado de equilíbrio. Dessa forma, o risco correspondente será o máximo risco possível para aquele contaminante, mesmo que demore algum tempo para ser observado.

Em termos de avaliação de risco à saúde humana, essa informação é muito significativa, pois revela a intensidade da exposição e dos efeitos adversos até seu valor máximo, fornecendo mais segurança sobre as estimativas do risco. Adicionalmente, tais resultados demonstram as diferentes possibilidades a serem adotadas quando está sendo realizada uma avaliação de risco à saúde humana.

\section{CONCLUSÃO}

Este artigo teve o objetivo de verificar as implicações em utilizar as geometrias de plano vertical normal ao fluxo subterrâneo e do prisma nas estimativas do risco à saúde humana devido à ingestão de água subterrânea contaminada por benzeno. Algumas conclusões que podem ser destacadas são:

- a aplicação de diferentes geometrias de fonte não interfere consideravelmente nos resultados obtidos, sendo importante avaliar qual tipo se aplica melhor a cada caso;

- o fato de que a concentração de equilíbrio pode levar muito tempo para ser alcançada requer que sejam considerados os cenários de exposição presentes e futuros, bem como a futura utilização da área em análise;

- a diferença existente entre os tempos em que foram atingidas as concentrações de equilíbrio para ambos os casos pode estar relacionada à forma como os contaminantes se dispersam a partir da fonte de contaminação, considerando que no caso do prisma a dispersão acontece ao longo de todo o comprimento da fonte;
- os riscos carcinogênicos obtidos por uma e outra geometria não foram significativamente diferentes. Dessa forma, o prisma pode ser visto como uma forma de estimar o risco com um nível de segurança ainda maior. No entanto, isso não diminui a importância que se tem dado até hoje à geometria de plano vertical para a simulação do transporte de contaminantes na maioria dos casos.

Como sugestão para trabalhos futuros, indica-se uma análise mais profunda sobre como os contaminantes são liberados a partir da fonte de contaminação, com o intuito de verificar qual das duas geometrias representa melhor os aspectos físicos do transporte de contaminantes. Primeiro no que diz respeito aos termos advectivos nas equações, para os quais a velocidade e o retardo são os parâmetros mais importantes; e segundo no que se refere aos termos dispersivos, ou seja, aos coeficientes de difusão, uma vez que na equação do prisma aparece o termo F1, o qual incorpora, mais uma vez, os coeficientes de dispersão na quantificação da concentração.

\section{REFERÊNCIAS}

BATLE-AGUILAR, J.; BROUYÈRE, S.; DASSARGUES, A.; MORASCH, B.; HUNKELER, D.; HÖHENER, P.; DIELS, L.; VANBROEKHOVEN, K.; SEUNTJENS, P.; HALEN, H. Benzene dispersion and natural attenuation in a alluvial aquifer with strong interactions with surface water. Journal of Hydrology, v. 369, p. 305-317, 2009. 
CARTER, D. A.; HIRST, I. L.; MADDISON, T. E.; PORTER, S. R. Appropriate risk assessment methods for major accident establishments. Trans IChemE, v. 81, part B, p. 12-18, 2003.

COVELLO, V. T. Communicating risk in crisis and noncrisis situations: tools and techniques for effective environmental communication. In: KOLLURU, R. V.; BARTELI, S.; PITBLADO, R.; STRICOTT, S. (Eds.). Risk assessment and management handbook for environmental, health and safety professionals. New York: McGraw-Hill, 1996. p. 15.3-15.44.

DOMENICO, P. A. An analytical model for multidimensional transport of a decaying contaminant species. Journal of Hydrology, v. 91, p. 49-58, 1987.

GUYONNET, D.; NEVILLE, C. Dimensionless analysis of two analytical solutions for 3-D solute transport in groundwater. Journal of Contaminant Hydrology, v. 75, p. 141-153, 2004.

KOLLURU, R. V. Health risk assessment: principles and practices. In: KOLLURU, R. V.; BARTELI, S.; PITBLADO, R.; STRICOTT, S. (Eds.). Risk assessment and management handbook for environmental, health and safety professionals. New York: McGraw-Hill, 1996. p. 4.3-4.68.

LEIJ, F. J.; PRIESACK, E.; SCHAAP, M. G. Solute transport modeled with Green's functions with application to persistent solute sources. Journal of Contaminant Hydrology, v. 41, p. 155-173, 2000.

MASSABÓ, M.; CIANCI, R.; PALADINO, O. Some analytical solutions for two-dimensional convection-dispersion equation in cylindrical geometry. Environmental Modelling \& Software, v. 21, p. 681-688, 2006.

McCALLUM, D. B.; SANTOS, S. L. Participation and persuasion: a communication perspective on risk management. In: KOLLURU, R. V.; BARTELI, S.; PITBLADO, R.; STRICOTT, S. (Eds.). Risk assessment and management handbook for environmental, health and safety professionals. New York: McGraw-Hill, 1996. p. 6.1-16.32.

MELO, T. M. Soluções analíticas para transporte de hidrocarbonetos do petróleo em água subterrânea: avaliação determinística e probabilística do risco à saúde humana. $154 \mathrm{f}$. Dissertação (Mestrado em Recursos Hídricos e Saneamento Ambiental) - Universidade Federal do Rio Grande do Sul, Porto Alegre, 2010.

NATIONAL RESEARCH COUNCIL - NRC. Commission on Life Sciences. Board on Environmental Studies and Toxicology. Committee to Review the OMB Risk Assessment Bulletin. Scientific Review of the Proposed Risk Assessment Bulletin from the Office of Management and Budget. Washington: National Academy Press, 2007. 286 p.

NATIONAL RESEARCH COUNCIL - NRC. Commission on Life Sciences. Committee on the Institutional Means for Assessment of Risks to Public Health. Risk assessment in the Federal Government: managing the process. Washington: National Academy Press, 1983. 191p.

ÖRBEG, T.; BERGBÄCK, B. A review of probabilistic risk assessment of contaminated land. Journal of Soils \& Sediments, v. 5, n. 4, p. 213-224, 2005.

PARK, E.; ZHAN, H. Analytical solutions of contaminant transport from finite one-, two-, and three-dimensional sources in a finite-thickness aquifer. Journal of Contaminant Hydrology, v. 53, p. 41-61, 2001.

RICE, G.; McDONELL, M.; HERTZBERG, R. C.; TEUSCHLER, L.; PICEL, K.; BUTLER, J.; CHANG, Y.; HARTMANN, H. An approach for assessing human exposures to chemical mixtures in the environment. Journal of Toxicology and Pharmacology, $v$. 233, p. 126-136, 2008.

SAGAR, B. Dispersion in three dimensions: approximate analytical solutions. Journal of Hydraulics Division, v. 108, p. 47-62, 1982.

SCHLECHTER, W. Process risk assessment - using science to "do it right". International Journal of Pressure Vessels \& Piping, v. 61, p. 479-494, 1995. 
SIM, Y.; CHRYSIKOPOULOS, C. V. Analytical solutions for solute transport in saturated porous media with semi-infinite or finite thickness. Advances in Water Resources, v. 22, n. 5, p. 507-519, 1999.

TAKEUCHI, E. Aplicação da metodologia RBCA para o município de Porto Alegre: definição de níveis aceitáveis baseados no risco para hidrocarbonetos. 157 f. Dissertação (Mestrado em Geociências) - Universidade Federal do Rio Grande do Sul, Porto Alegre, 2008.

UNITED STATES ENVIRONMENTAL PROTECTION AGENCY - USEPA. Risk Assessment Guidance for Superfund. Human Health Evaluation Manual (Parte A). USEPA, 1989. v. 1.

WORLD HEALTH ORGANIZATION - WHO. International Program on Chemical Safety (IPCS): IPCS risk assessment terminology. Genebra: WHO, 2004. 